Revista de Psicología de la PUCP. Vol. XVII, 2, 1999.

\title{
SITUACIÓN ACTUAL DE LA ENSEÑANZA DEL PSICODIAGNÓSTICO DE RORSCHACH Y LOS MÉTODOS PROYECTIVOS EN EL PERÚ 1
}

\author{
Matilde Ráez de Ramírez ${ }^{2}$ \\ Pontificia Universidad Católica del Perú
}

Se presenta una breve historia sobre el origen y desarrollo de las pruebas psicológicas proyectivas en el P'erú. Se administró una encuesta a profesores universitarios y a miembros de la Sociedad Peruana de Rorschach y Métodos Proyectivos, con el propósito de conocer la situación actual de la enseñanza de pruebas. Los resultados demuestran que existe una corriente de apertura hacia el pensamiento científico, con énfasis en la investigación y en el empleo de pruebas estandarizadas. Asimismo se muestra que el marco conceptual de trabajo está basado en los cambios actuales en psicología.

Palabras claves: Rorschach, pruebas poyectivas de personalidad, enseñanza y entrenamiento, investigación, marco conceptual, cambios significativos en procedimientos, rol del psicólogo.

The present situation about the teaching of Rorschach and other proyective tests in Peru This paper presents a brief history about the origin and development of the proyective techniques in Peru. A questionnaire was administered to universitiy professors and members of the Peruvian Rorschach and Proyective Methods Society in order to collect data concerning the test training situation. Results indicate that there is a broad vicw towards scientific thought that stresses research and the use of standardized tests. It is also pointed out, that the conceptual frame work is based on current changes in psychology.

Key words: Rorschach, proyective techniques, teaching and training, research, conceptual frame, procedural significant changes, psychologist role.

1. Una versión del presente trabajo fue presentado en el Simposium sobre "Enseñanza y entrenamiento del Rorschach y de los Mérodos Proyecrivos" en el XV Congreso Internacional de Rorschach y Métodos proyectivos, (Amsterdam, Holanda, 1999).

2. Doctora en psicología por la Universidad de Nimega (Holanda). Psicóloga clínica: Práctica profesional, docencia e investigación. Profesora Principal de la Pontificia Universidad Católica del Perú. Profesora del los cursos de "Pruepas proyectivas de personalidad", "Psicodiagnóstico de Rorschach" y "Diagnóstico de Adultos". Presidenta y Miembro fundador de la "Sociedad Peruana de Rorschach y Métodos Proyectivos". Autora de libros y numerosos artículos sobre el tema. Correo electrónico: mraez@pucp.edu.pe. 

La psicología peruana en el campo de las pruebas tiene una tradición clínica significativa. Fue el propio Hermann Rorschach quien envió en 1920 sus láminas al profesor $\mathrm{H}$. Delgado, eminente psiquiatra peruano, y en 1924 aparece el primer trabajo de Rorschach en la literatura médica peruana: "La aplicación del test de interpretación de Formas". En 1927 Delgado introduce la técnica en el campo forense con un estudio a ciegas sobre un delincuente juvenil (Ráez, 1996).

Blumenfeld, en 1935, creó en Lima una escuela de psicometría con los últimos avances del pensamiento científico. Es así que el área de la psicometría y también de las técnicas proyectivas tuvo un importante desarrollo hasta los sesentas, con el empleo de pruebas adaptadas a nuestra realidad. Lamentablemente al morir Blumenfeld y debido a problemas políticos, se cerró el instituto.

Los setentas y ochentas se caracterizaron por la violencia terrorista y el trabajo aislado en las universidades, que con mucho esfuerzo mantuvieron el nivel académico y los vínculos con la comunidad científica internacional. Sin embargo Lazo y Zanolo (1996) en una valiosa recopilación de datos sobre investigación en el Perú señalaron la existencia hasta 1991, de 1638 tesis de bachillerato de investigación empírica con pruebas, demostrando que a pesar de las dificultades existe mucho interés en el tema. También, los trabajos presentados en los Congresos de Psicología resaltaron el interés en la investigación, especialmente en las áreas clínica y de diagnóstico.

Felizmente en la década de los noventa al desaparecer el terrorismo, se amplió el intercambio de conocimiento con otros centros de desarrollo psicológico y se abrió una nueva perspectiva para la enseñanza y utilización de las pruebas psicológicas. 
Este estudio se centra en la situación actual de las pruebas proyectivas en el Perú. Está dividido en dos partes:

- La primera se refiere a la situación actual de la enseñanza y empleo de los métodos proyectivos y sus perspectivas futuras en el Perú.

- La segunda se refiere al marco conceptual y modelo de trabajo con pruebas, tomando en cuenta los cambios significativos que se están produciendo en la ciencia psicológica.

La carrera de psicología en el Perú cuenta con aproximadamente 5,153 estudiantes. Se enseña en 15 universidades, nueve de ellas en la capital y seis en provincias. En Lima, dos son nacionales y siete son privadas. Sólo en una universidad privada no se enseñan los cursos sobre pruebas proyectivas (Anicama, 1999).

\section{Metodología}

Se aplicó en 1999, una encuesta en rodas las universidades de Lima en las que se enseñan técnicas proyectivas y a los directivos de la Sociedad Peruana de Rorschach y Métodos Proyectivos, responsables de los cursos de especialización con pruebas.

En el Cuadro 1 se presentan los datos de los participantes. Estos son 31 , de los cuales 25 son mujeres y 6 son hombres. Ellos tienen los grados universitarios de doctores, magisters, licenciados y bachilleres. El $42 \%$ tiene más de 20 años de docencia en contraste con el $26 \%$ que tiene de 1 a 4 años. Es interesante observar la combinación entre profesionales con una amplia experiencia en el campo y los jóvenes que se inician. 
Situación actual de la enseñanza del psicodiagnóstico de Rorschach...

Cuadro 1

Distribución de la muestra

\begin{tabular}{|l|l|l|}
\hline Variable & n & $\%$ \\
\hline Sexo & 25 & 81 \\
Femenino & 6 & 19 \\
Masculino & & \\
Grado Universitario & 6 & 19 \\
Bachiller & 7 & 23 \\
Licenciado (a) & 13 & 42 \\
Magister & 5 & 16 \\
Doctor (a) & & \\
Tiempo de práctica con pruebas & 13 & 42 \\
Más de 20 años & 6 & 19 \\
De 10 a 20 años & 4 & 13 \\
De 5 a años & 8 & 26 \\
De 1 a 4 años & & \\
\hline
\end{tabular}

\section{Resultados y Discusión}

Para facilitar la lectura, los resultados se presentan para cada una de las partes del estudio.

1. La situación actual de la enseñanza y empleo de los métodos proyectivos en el Perú

\section{Enseñanza de pruebas de personalidad}

En relación con auto informes y cuestionarios se encontró que MMPI y Eysenck son los más empleados (25.8\%). Un dato interesante es la 
amplia gama tanto de las nuevas como de las antiguas técnicas en el área (Figura 1).

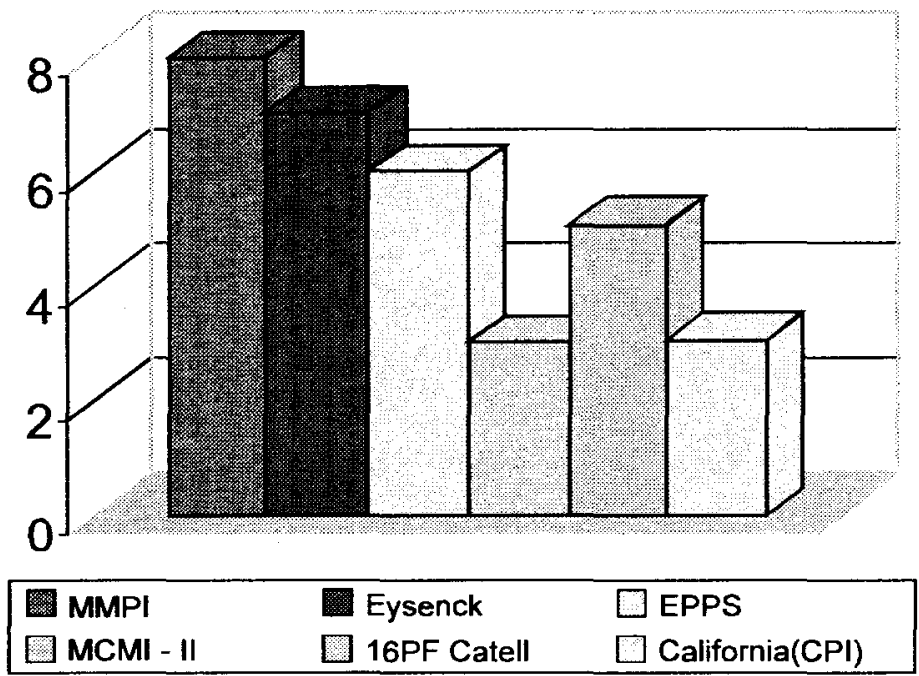

Figura 1. Inventarios y Autoinforme

El test de Rorschach siguiendo el Sistema Comprehensivo de Exner es el más utilizado (35.5\%) (Figura 2). Este dato indica un cambio en la orientación docente, porque en el estudio que realizamos en 1996 para el Congreso Mundial de Psicología de Montreal, sólo el 15\% lo utilizaba. Pensamos que se debe al hecho que la mayoría de estudios en la comunidad científica en la actualidad se. llevan a cabo con este sistema. En contraste, sólo 3 (el 9\%) enseñan el sistema de Klopher y ninguno el de Bohm. 
Situación actual de la enseñanza del psicodiagnóstico de Rorschach...

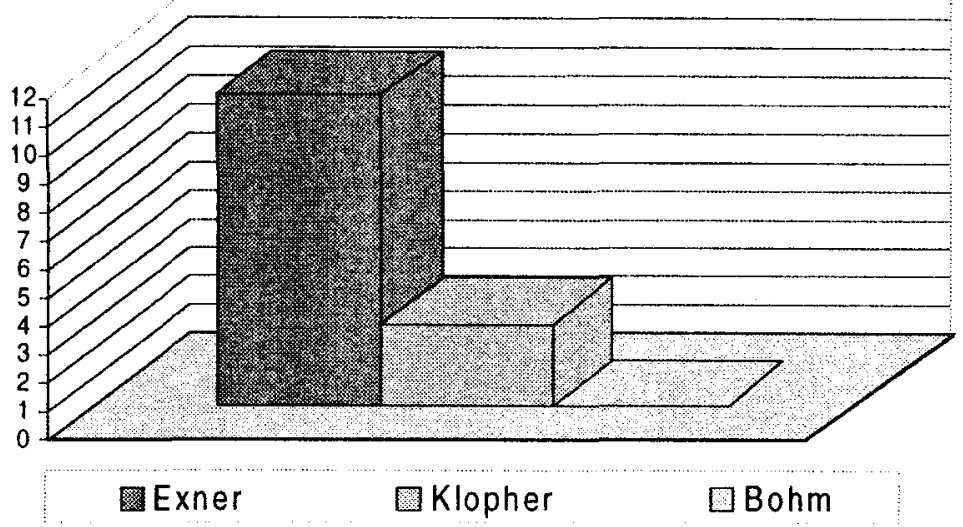

Figura 2. Psicodiagnóstico Rorschach

En relación con T.A.T (Figura 3) la técnica de Shentoub que se introdujo en 1990, es el método más usado. Esto indica el interés por estudios de la personalidad más en profundidad.

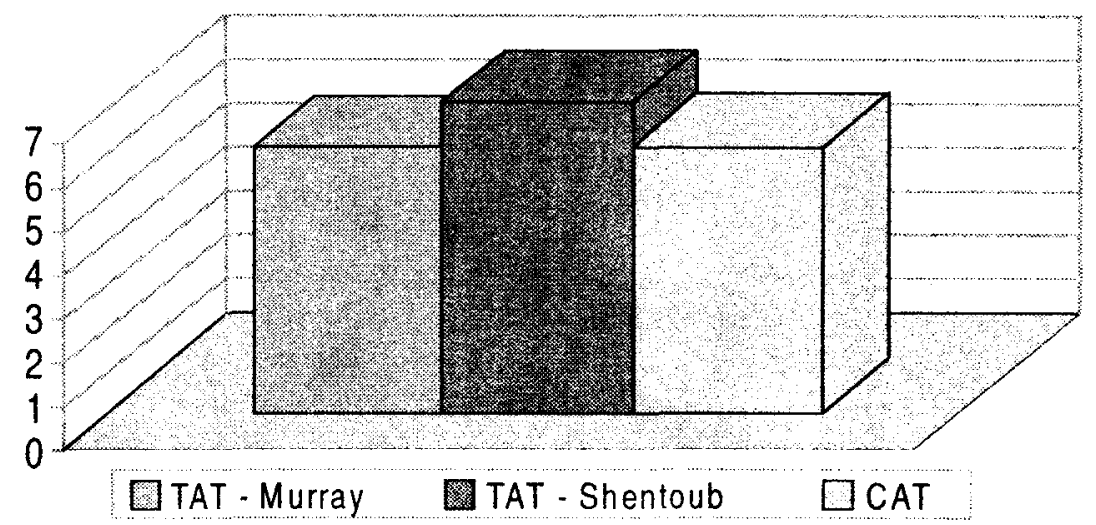

Figura 3. Test de Apercepción Temática 
Entre los Métodos Proyectivos de Completamiento (Figura 4) destacan las pruebas de completamiento de frases, siendo el Sacks enseñada por más de la mitad de los docentes (51.6\%), seguido por Tres Personajes de Backes-Thomas y el Desiderativo (22.6\%). Completud de Frases es una prueba valiosa por la variedad de datos que proporciona y además por la facilidad de su administración y el bajo costo de material empleado.

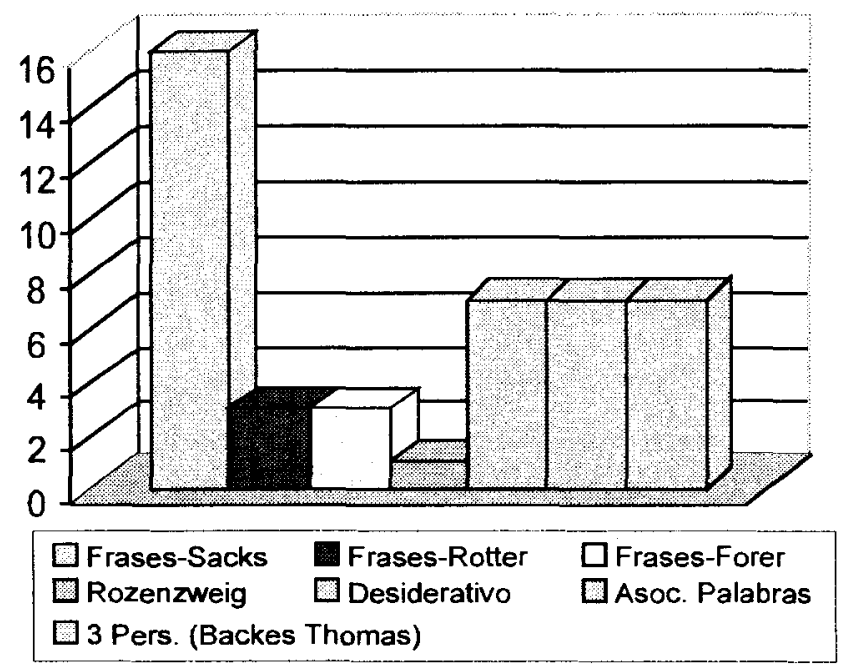

Figura 4. Test proyectivos, asociativos y de completamiento.

Las pruebas gráficas (Figura 5) son las más enseñadas (58.1\%). Esto se debe, entre otras razones, a que han demostrado su utilidad a lo largo de muchos años. Además su administración es sencilla y el material es de bajo costo. Resultan valiosas tanto para estudios de adultos (Machover, especialmente) como de niños (Familia que es la prueba más usada).

Un porcentaje menor de docentes (7\%), utiliza pruebas más especializadas y que se refieren al ámbito infantil, tales como la Hora de Juego. 
Situación actual de la enseñanza del psicodiagnóstico de Rorschach...

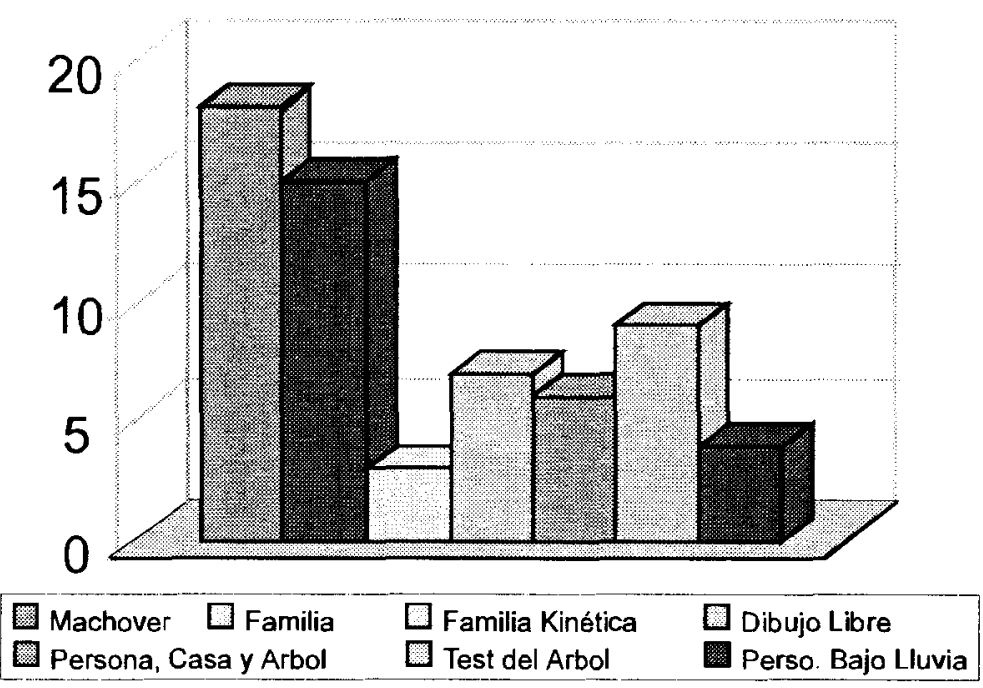

Figura 5. Pruebas Proyectivas Gráficas.

Una inferencia interesante a partir de estos cuadros es que el número de docentes que enseñan pruebas proyectivas supera a los que enseñan tests objetivos de personalidad, lo cual indica la importancia de los métodos proyectivos, en el ámbito docente universitario.

La variedad de las técnicas enseñadas señala que además de la influencia de la escuela norteamericana, los docentes también mantienen vínculos con los países francófonos (T.A.T. de Shentoub, Tres Personajes, entre otros) y con la Argentina (Desiderativo).

En la Figura 6, se presentan las principales dificultades para el empleo y la enseńanza de las pruebas. Se ha utilizado los índices de frecuencia ponderando los rasgos de preferencia de uso con constantes lineales. Se ha encontrado que la mayor limitación es económica. Se explica que "la falta de normas para el país" y "el escaso material de pruebas" se debe al hecho de "no tener ni tiempo, ni dinero" para cumplir estos objetivos. 


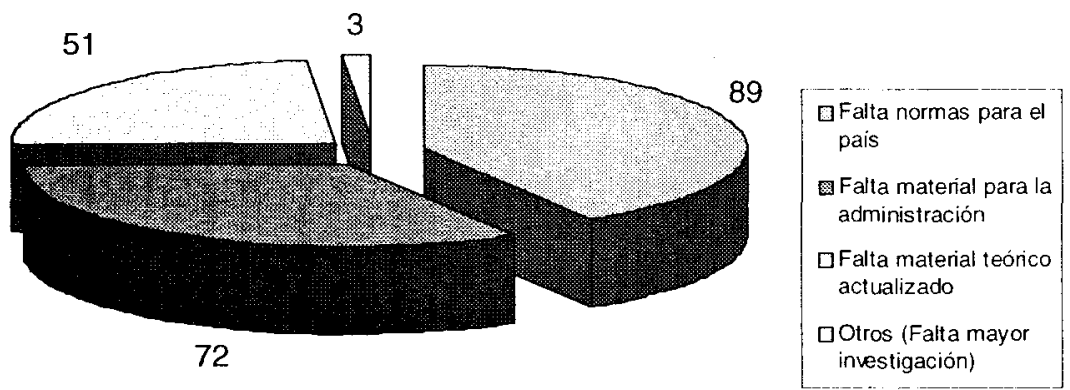

Figura 6. Dificultades para uso y enseñanza de Test de Personalidad.

Referente a la necesidad de emplear técnicas proyectivas, el $62 \%$ considera que son "totalmente necesarias" para la formación de los psicólogos y ninguno las considera innecesarias (Figura 7). El hecho que todos son docentes del área indica su experiencia respecto al valor de las técnicas y a su uso en un país pluricultural como el nuestro.

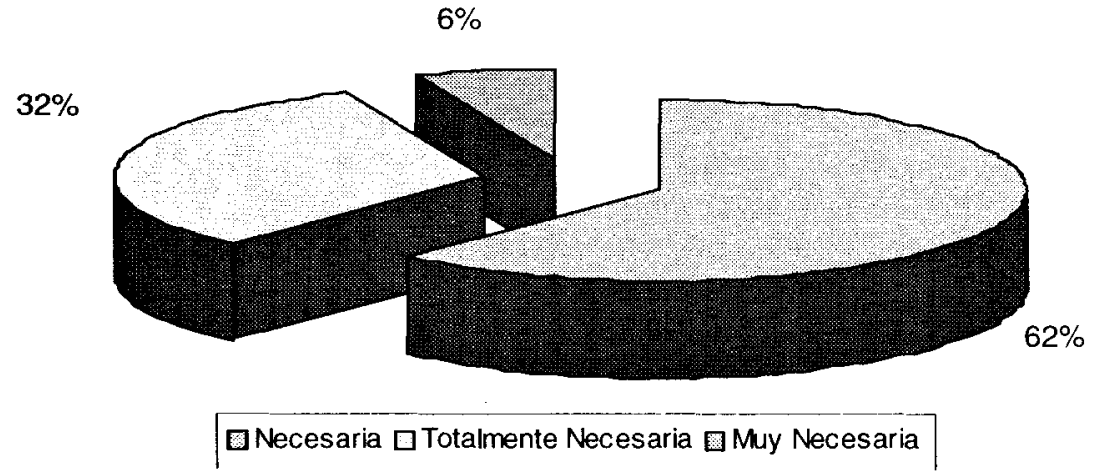

Figura 7. Necesidad de enseñanza de Técnicas Proyectivas. 
Situación actual de la enseñanza del psicodiagnóstico de Rorschach...

Las corrientes teóricas más usadas son la Psicoanalítica $(70 \%)$ y la Humanista (16\%) (Figura 8). Obviamente esta tendencia se debe a que el marco teórico de los tests proyectivos es básicamente dinámico.

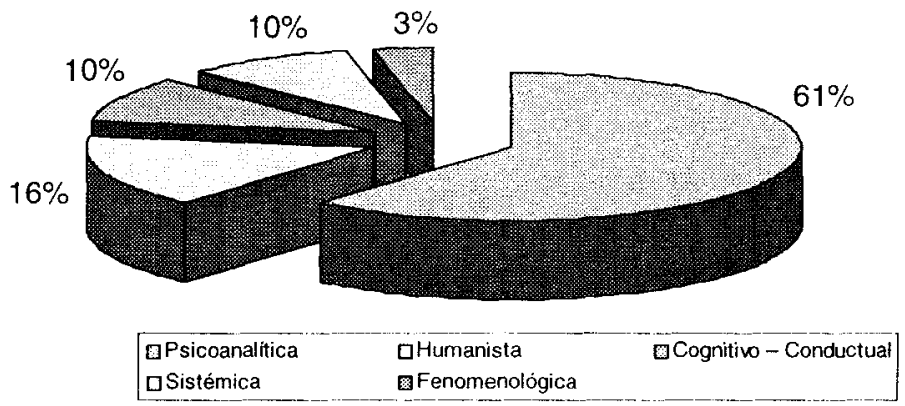

Figura 8. Corrientes teóricas usadas en trabajo con Técnicas Proyectivas.

\section{Uso de las pruebas en la práctica profesional}

El $84 \%$ utilizan método proyectivos en su práctica profesional, manteniendo así coherencia en el rol. Los que no las usan son algunos de los que enseñan pruebas objetivas. Sólo un participante que enseña métodos proyectivos no los emplea en su trabajo diario (Figura 9).

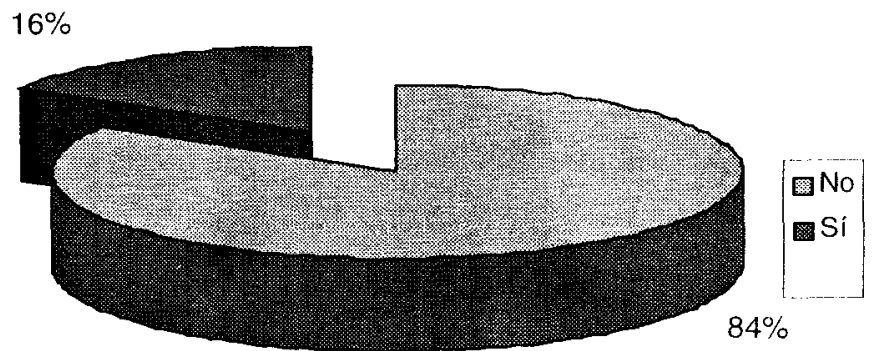

Figura 9. Uso de Técnicas Proyectivas en Práctica Profesional. 
De los tests proyectivos, el Rorschach es la prueba más usada, seguida de las técnicas gráficas y los tests temáticos (Cuadro 2). Su valor es apreciado por los expertos e incluso la emplean la mayoría de los que no la enseñan.

Cuadro 2

Técnicas Proyectivas Usadas con Mayor Frecuencia

\begin{tabular}{|c|c|c|c|c|c|c|c|c|c|}
\hline 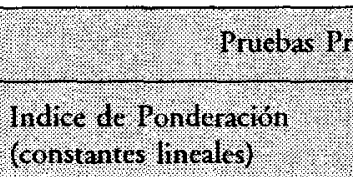 & 8 & $\frac{1}{4} .7$ & 6 & 5 & 4 & 3 & 2 & 1 & $f$ \\
\hline Rorschach & 18 & 4 & 1 & & & & & & 178 \\
\hline Gráficas & 5 & 11 & 6 & 2 & & & & & 163 \\
\hline TAT/CAT & & 8 & 4 & 2 & 1 & & & 1 & 95 \\
\hline Completamiento de Frases & & 1 & 7 & 5 & & 1 & & & 77 \\
\hline Desiderativo & & & 2 & 3 & 1 & 1 & & & 34 \\
\hline Asociación de Palabras & & & & 1 & 4 & 3 & & & 30 \\
\hline Colores & & & 1 & & 1 & 1 & 1 & & 15 \\
\hline Tres Personajes & & & & 2 & & & & & 10 \\
\hline Phillipson (TRO) & & & & & 2 & & & & 8 \\
\hline Rosenzweig & & & & & & & 1 & & 2 \\
\hline
\end{tabular}

Nota. "Los índices de frecuencia son calculados ponderando los rangos de preferencia de uso con constantes lineales.

En el Cuadro 3 se presentan todos los instrumentos de evaluación no proyectivos que utilizan los docentes. Un dato interesante lo constituye el hecho que sean los índices de inteligencia de Wechsler, las pruebas más usadas, seguidos del Bender y del MMPI. Además es significativa la variedad de cuestionarios que se utilizan indicando la amplitud de los intereses y del campo de aplicación, así como la actualización de los profesores. 
Situación actual de la enseñanza del psicodiagnósrico de Rorschach...

Cuadro 3

Otros Instrumentos de Evaluación Usados con Mayor Frecuencia

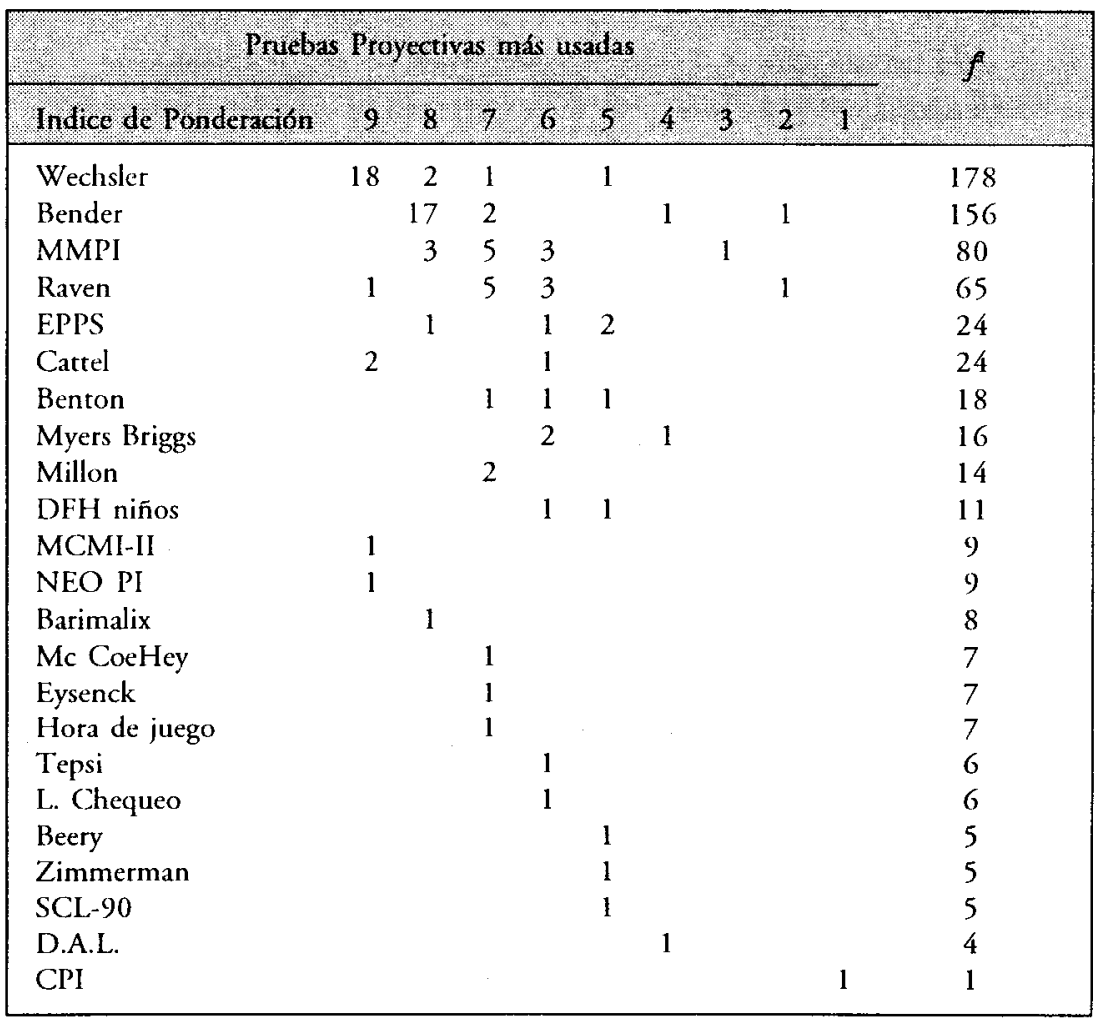

Nota. " Los índices de frecuencia son calculados ponderando los rangos de preferencia de uso con constantes lineales. 


\section{Investigación}

Por su especial significado la hemos incluido en un rubro aparte.

El $90 \%$ de los encuestados ha llevado a cabo investigaciones con pruebas. Este constituye un hallazgo valiosos, pues prácticamente todos los docentes investigan sobre su área. Sólo el 9.7, tres docentes, nunca lo han hecho.

Los estudios llevados a cabo son sus tesis de bachilleres, de maestría, de doctorado y también investigaciones específicas sobre temas y pruebas que les interesan para su trabajo diario, o para profundizar algún asunto en especial.

Tres docentes han escrito libros sobre pruebas y algunos señalan que editan manuales de trabajo interno para sus alumnos, pero que no se publican como libros por la falta de apoyo económico de la universidad.

En el Cuadro 4 se presentan las pruebas proyectivas más investigadas. Nuevamente el Rorschach con un significativo $48 \%$ es la más utilizada, seguida del Bender y el Benton, ambas pruebas viso motoras y el Cattell con el $9.7 \%$. Esto se relaciona con el hecho que a partir de los 90, que se introdujo el Sistema Comprehensivo en el país, el test de Rorschach está resultando una técnica estimulante para investigar, porque al favorecer el empleo de los métodos más utilizados por la comunidad científica, unifica el lenguaje y favorece su expansión. Llama la atención en el caso opuesto, que siendo las escalas de Wechsler las técnicas más usadas, sean las menos investigadas. Quizá se deba al hecho que un estudio normativo exige un esfuerzo de trabajo en equipo, ingentes recursos económicos y prácticamente dedicación exclusiva. Todas estas exigencias resultan muy difíciles de ser cubiertas en el país.

Las mayores limitaciones para investigar, pregunta abierta donde se podían señalar una o varias razones, enfatiza nuevamente, la falta de normas estandarizadas y también falta de financiamiento $(51 \%)$, en tanto 
Situación actual de la cnseñanza del psicodiagnóstico de Rorschach...

Cuadro 4

Pruebas Utilizadas en la Investigación

\begin{tabular}{|lcc|}
\hline Pruebas usadas en investigaciones Clinicas & $f$ & 8.0 \\
\hline Rorschach & 15 & 48,4 \\
Bender & 3 & 9,7 \\
Benton & 3 & 9,7 \\
Catell & 3 & 9,7 \\
MMPI & 2 & 6,5 \\
Test de Familia & 2 & 6,5 \\
Eysenck & 2 & 6,5 \\
Spielberg & 2 & 6,5 \\
T.A.T. & 1 & 3,2 \\
Complet. de frases & 1 & 3,2 \\
MCMI-II & 1 & 3,2 \\
Escalas Weschler & 1 & 3,2 \\
PMA & 1 & 3,2 \\
Beck (Depresión) & 1 & 3,2 \\
Krow (índice clínico) & 1 & 3,2 \\
Raven & 1 & 3,2 \\
D.F.H. & 1 & 3,2 \\
\hline
\end{tabular}

que falta de material original de las pruebas vs. material "pirata" y "dificultad de acceso a muestras representativas" es señalado por el $16 \%$.

En relación a proyecciones futuras con las pruebas (Cuadro 5) que es una pregunta abierta, el $22 \%$ se refiere a obtener baremos de la realidad peruana, el $16 \%$ que se fomente la investigación y $9 \%$ mayor conocimiento de los marcos teóricos y mayor difusión de pruebas nuevas en el medio.

Es interesante el rango de opciones que indica un enfoque de apertura hacia las pruebas dentro del ámbito científico y preciso de las normas; la búsqueda de nuevas posibilidades actualizando las técnicas antiguas e introduciendo nuevas pruebas. Es posible que a través de esta variedad se representa la posición actual de la enseñanza de las pruebas y su apertura hacia el conocimiento actualizado. 
Cuadro 5

Proyecciones Futuras sobre Pruebas de Personalidad

\begin{tabular}{|c|c|c|}
\hline Principales limitaciones: & $f$ & $\%$ \\
\hline Es necesario obtener baremos para nuestra rea- & & \\
\hline lidad & 7 & 22,6 \\
\hline Se necesita que se fomente la investigación & 5 & 16,1 \\
\hline $\begin{array}{l}\text { Se necesita un mayor conocimiento de la teo- } \\
\text { ría de la prueba para su buen uso }\end{array}$ & 3 & 9,7 \\
\hline $\begin{array}{l}\text { Se necesita una mayor difusión de las pruebas } \\
\text { nuevas }\end{array}$ & 3 & 9,7 \\
\hline $\begin{array}{l}\text { Se necesita verificar su validez y confiabilidad } \\
\text { en nuestro medio }\end{array}$ & 2 & 6,5 \\
\hline Se necesita actualizar el material disponible & 2 & 6,5 \\
\hline $\begin{array}{l}\text { Se necesita crear pruebas que se adapten a } \\
\text { nuestro medio }\end{array}$ & 2 & 6,5 \\
\hline Es necesario validar las pruebas proyectivas & 1 & 3,2 \\
\hline
\end{tabular}

\section{Marco Conceptual y Modelo de Trabajo con Pruebas}

Plantearemos brevemente el marco conceptual y el modelo de trabajo que se llevó cabo en el ámbito de las pruebas proyectivas. Se indica los ejes teóricos y los cambios más significativos en los procedimientos, campo de trabajo y rol del psicólogo en el área.

El uso actual de los tests señala modificaciones sustanciales. Se deja de lado la fase tecnocrática y se focaliza el interés en una estructura y un concepto holístico, es decir en la mutualidad entre el ambiente y la conducta.

Uno de los cambios más significativos es el que se refiere a la importancia práctica del concepto de personalidad y el empleo de las teorías de personalidad con los modelos teóricos para la evaluación. 
Situación actual de la enseñanza del psicodiagnóstico de Rorschach...

Como consecuencia, los procedimientos utilizados también presentan algunos cambios relevantes y la revalorización se encuentra de manera explícita en una variedad de áreas.

\section{En el campo del trabajo profesional}

- Dentro de este enfoque se incluye el trabajo con no pacientes, es decir, estudios de la personalidad dentro del campo de la Psicología del Desarrollo, con énfasis en las distintas etapas que constituyen el Ciclo Vital (Life-Span).

- En el Perú, la mayoría de profesionales en la práctica privada o en psicología de la salud, trabajan en consultorios externos. Generalmente los motivos de consulta se refieren al estrés, a insuficiencias sociales, a conflictos interpersonales, con síntomas como la ansiedad o la depresión; pero en realidad se refieren como elemento unificador a conductas desadaptadas, enraizadas en el comportamiento, sentimientos, pensamientos, es decir, "en su estilo de personalidad". Éste es el eje a partir del que se desenvuelve el modelo de trabajo con pruebas.

\section{Modificaciones conceptuales en el DSM-IV}

- Los síndromes de personalidad ocupan un lugar destacado, reflejando los cambios que se han señalado y constituyendo así el aspecto central del sistema multi-axial.

- Lo esencial es que la personalidad es útil como el sustrato dinámico a partir del cual se capta el sentido de los trastornos, sean floridos o transitorios, así como de los procesos y de las crisis del desarrollo evolutivo y se les ubica en un papel contextual que los hace fundamentales en la comprensión e interpretación de otras patologías, o de procesos esperados del desarrollo evolutivo. 
Cambios más significativos en los procedimientos relacionados con los avances tecnológicos y las modificaciones conceptuales teóricas

- Desarrollo de técnicas más centradas en el campo en estudio y que han alcanzado mayor confiabilidad y validez, enriqueciendo sus propiedades psicométricas. En este punto se señala especialmente la introducción del MMPI-2, del Sistema Comprehensivo de Exner y del Inventario Clínico Multiaxial de Millon.

- Mayor confianza en las interpretaciones específicas y no en las generalizaciones absolutas anteriores.

- Mayor interés en los factores situacionales y en los ambientales como determinantes de la conducta. Énfasis en el presente que es donde el pasado está actualmente operativo y donde influencia el futuro. Lo ideal es la comprensión del presente que incluye el conocimiento de cómo se ha evolucionado.

Cambios en los campos de trabajo relacionados con los avances de la ciencia $y$ las exigencias de la sociedad

- Los notables adelantos en el estudio e investigación del cerebro han revolucionado el estudio y la evaluación en el campo de las Neurociencias. En la actualidad existe un mayor conocimiento del sistema nervioso en general y de las relaciones entre funciones específicas del cerebro y la conducta. Se utiliza la evaluación neuropsicológica para determinar tanto los cambios generales, como el funcionamiento específico en áreas intelectuales y emocionales, en pacientes que han sufrido traumatismo cerebral, en epilepsia y en la variedad de procesos alterados como reacción a las drogas. Las pruebas más comunes son las Escalas de Wechsler, Bender y Benton. El Rorschach y el T.A.T. se utilizan siguiendo parámetros muy específicos. Desaparecen así los estudios sobre pacientes orgánicos.

- La creciente importancia de áreas tales como la evaluación ambiental, el modelo ecológico. Ya Lewin indicaba que la conducta 164 
Situación actual de la enseñanza del psicodiagnóstico de Rorschach...

estaba en función de la persona y el entorno. En este modelo ecológico es de gran importancia para la evaluación, estudiar directamente la naturaleza de los entornos, tanto en forma general, como en la forma particular en que afecta a los clientes. Se tiene en cuenta el sistema social y los sistemas más pequeños como la familia y sus grupos de referencia. Se emplean el Rorschach Consensus y el Test de la Familia.

- Las modificaciones de modelos y métodos en la evaluación infantil.

Cambios en el rol y asunción de una nueva ubicación laboral. El psicólogo deviene en un consultor

- Los psicólogos peruanos en general y los docentes universitarios en particular, estamos luchando porque desaparezca la concepción de "administrador", de "técnico" para dar paso al Consultor. El evaluador es un consultor. No se trata sólo de cambio de nombre, sino de modificar el afronte ante la tarea y el enfoque del estudio y también implica un cambio en la percepción de sí mismo del Psicólogo Consultor.

- Se explica el cambio a través de un ejemplo. En muchos servicios de salud el sistema establecido implica pedir al psicólogo una prueba específica. Puede ser un test de personalidad, incluso algunos señalan Rorschach o Figura Humana. Un psicólogo "técnico" toma el Rorschach, señala características, quizá indique algún dato puntual, tal vez añade alguna otra prueba y entrega su informe dentro de este procedimiento.

- El consultor pide a quien le envió el caso que amplíe su pedido y se interesa en saber dentro de qué contexto se da la demanda y cuáles son realmente las expectativas de dicha demanda. Recién, entonces, organiza la evaluación dentro de su esquema y por supuesto de acuerdo a la persona que evalúa. Puede o no urilizar las pruebas que se le pidieron, mas su informe está abriendo otras posibilidades no pensadas al que lo pidió. 
- Se intenta, así, cambiar el concepto que el trabajo requiere una aceptación acrítica para mantener una tradición intra e interprofesional. Se pone el bienestar del cliente en primer lugar, con confianza en nuestros procedimientos, confianza que viene en parte de un cuestionamiento periódico de los avances. En el ejemplo señalado se interesa al equipo de trabajo respecto a cómo el Rorschach utiliza las Constelaciones; es decir, los nuevos datos del sistema en relación con elementos psicopatológicos; los signos especiales que se refieren a los elementos más sutiles de la personalidad y su valor psicométrico para mejorar la predicción del estudio.

En conclusión, podemos señalar algunos aspectos centrales de la investigación:

Se enfariza el conocimiento técnico de construcción de pruebas con información actualizada sobre los estudios de validez y de normatividad. Resulta esencial el empleo de los distintos significados de los puntajes y de los fenómenos específicos.

Se postula que el uso debe ser llevado a cabo por expertos. El material de las pruebas es útil, por ejemplo, para medidas comparativas de niveles de logros, o para observar las formas en las que la persona responde a diversas tareas y encontrar las oportunidades para intentar caminos alternativos. Actualmente con las pruebas lo que se intenta, sobre todo, es proporcionar instancias del comportamiento. Los tests son sustitutos eficientes para comprender al individuo a través de una variedad de conductas.

Trabajamos para evitar el empleo rutinario de las pruebas en el que se pierde tiempo, energía y dinero. El objetivo final es utilizarlas de acuerdo a la demanda del caso.

También es imprescindible el uso de un marco referencial que se apoye en las teorías psicológicas actuales. Asimismo, se refuerza el método científico con la validez y confiabilidad de las técnicas en uso. 
Situación actual de la enseñanza del psicodiagnóstico de Rorschach...

\section{Referencias}

Anicama, J. (1999). La psicología en el Perú. En M. Alonso (Ed.) y Eagly, A. (Ed. Asoc.), Psicologia en las Américas. Caracas: Sociedad Interamericana de Psicología.

Lazo, J. y Zanollo, B. (1996). Investigación y Publicaciones Psicológicas en el Perú. Lima: San Marcos.

Ráez de Ramírez, M. (1994). Rorschach's Psychodiagnosis in Peru. Rorschachiana, 19, 146-155.

Ráez de Ramírez, M. (1996). Las pruebas psicológicas en el Perú. Trabajo presentado en el XXVI Congreso Internacional de Psicología, Montreal.

Ráez de Ramírez, M. (1998). La ubicación, la enseñanza y el desarrollo de las pruebas proyecrivas en el Perú. Trabajo presentado en el $\mathrm{X}$ Congreso Latinoamericano de Rorschach y otras técnicas Proyectivas, Santiago de Chile. 